\title{
La comparaison comme politique: le tournant du début du $21^{e}$ siècle
}

\section{António Nóvoa, Luís Miguel Carvalho et Cristina Yanes}

Notre texte se pose en essai, libre, cherchant à ouvrir un débat d'idées bien plus qu'à démontrer empiriquement certaines thèses. L'argument central que nous y développons concerne l'émergence, à partir de l'an 2000, date symbolique que nous avons retenue, d'une série de changements majeurs dans la manière dont les "données internationales et comparées» sont incorporées dans les politiques éducatives nationales ou, autrement dit, dans la manière dont ces données induisent des politiques de traduction qui se distinguent, très nettement, de celles qui ont caractérisé le $20^{\circ}$ siècle.

\section{Introduction}

La comparaison est devenue l'un des plus puissants instruments d'action politique. Tout au long du $20^{\mathrm{e}}$ siècle, l'influence des travaux de comparaison a considérablement marqué la manière de penser et d'agir dans le champ de l'éducation. Aucun pays n’a pu rester indifférent aux résultats présentés. De ce fait, l'apparition de logiques d'appropriation et de traduction de ces résultats dans les politiques nationales est devenue inévitable.

Au début du $21^{\mathrm{e}}$ siècle, un tournant encore difficile à discerner est cependant en train d'avoir lieu. À partir de l'an 2000, une «solidification» des processus de comparaison nait, qui se lit à travers l'apparition de conséquences plus concrètes, matérielles, dans l'espace des politiques nationales. Notre article cherche à identifier et à comprendre ce tournant dont les premiers signes commencent à être bien visibles.

Disons-le tout de suite: ce texte se pose en essai, libre, qui cherche à ouvrir un débat d'idées bien plus qu'à démontrer à l'aide d'arguments. Dans ce sens, il propose au lecteur une «intuition réfléchie» sans aspirer à épuiser le sujet ou même à établir un raisonnement basé sur des approches empiriques. Il prolonge les analyses produites par António Nóvoa à propos des politiques éducatives européennes et de la comparaison comme mode de gouvernance (Nóvoa, 2010; Nóvoa \& Lawn, 2002; Nóvoa \& Yariv-Mashal, 2003) et par Luís Miguel Carvalho à propos de PISA $(2011,2012,2014)$. 
L'argument central que nous développons ici concerne l'émergence, à partir de l'an 2000, année symbolique que nous avons retenue, d'une série de changements majeurs dans la manière dont les "données internationales et comparées» sont incorporées dans les politiques éducatives nationales ou, autrement dit, dans la manière dont ces données induisent des politiques de traduction qui se distinguent, très nettement, de celles qui ont caractérisé le $20^{\mathrm{e}}$ siècle.

De fait, nous sommes particulièrement intéressés par la notion de «traduction", telle qu'elle a été travaillée par Michel Callon (1986) ou Bruno Latour (1989). Ce qui nous attire, c'est la manière dont les connaissances produites par différents acteurs sont intégrées à travers un jeu social complexe d'actions et d'interactions dans les stratégies de pouvoir d'entités politiques et économiques agissant au plan international. C'est cette perspective spécifique qui porte notre réflexion.

Ce qui nous paraît le plus significatif dans le tournant que nous essayons d'identifier, c'est le fait que les données sur l'éducation tendent à intégrer des ensembles ou "agrégats» d'indicateurs qui servent à évaluer les pays et leurs politiques. Ces ensembles influencent les décisions internationales concernant chaque État comme ils influencent les organisations intergouvernementales et les agences, fonds et investisseurs privés. Dans ce processus, qui en est encore à ses débuts et, du coup, difficile à cerner dans toutes ses conséquences, les résultats des enquêtes comparées tendent à produire des conséquences concrètes, tangibles pour les populations comme pour les gouvernements.

Nous nous pencherons sur deux cas, dans la mesure où ils révèlent bien cette tendance: 1- l'initiative PISA, Programme for International Student Assessment, lancée par l'OCDE en 1997 et dont la première enquête a eu lieu, justement, en l'an 2000 (Schleicher, 2006); 2- l'agenda éducatif de l'Union Européenne tout particulièrement tel qu'établit après l'adoption de la Stratégie de Lisbonne en 2000.

La première partie de l'article est consacrée à une analyse historique portant sur le rôle tenu par les statistiques scolaires comparées dans l'émergence des États enseignants et dans la diffusion mondiale du modèle scolaire. Nous tenterons de repérer les métamorphoses qui caractérisent l'évolution de la raison statistique et comparée dès la fin du $19^{\mathrm{e}}$ siècle, avec le passage d'une "connaissance de l'autre» à une «mesure de l'autre» (Nóvoa \& Yariv-Mashal, 2003). Pour conclure, nous observerons que la popularité et la légitimité des statistiques comparées ainsi que leur impact sur les politiques nationales, ont depuis toujours été influencés par des logiques complexes de diffusion et de réception, d'exportation et d'importation, de proposition et d'appropriation.

Les deuxième et troisième parties de l'article analyseront deux réalités concrètes qui illustrent le tournant que nous voulons saisir et identifier: PISA et l'action de l'Union Européenne dans le domaine de l'éducation. Le premier cas, celui de PISA, permet d'identifier un renversement des logiques traditionnelles de récolte des données: au lieu de rassembler et de systématiser les informations recueillies dans les différents pays, PISA structure et centralise des procédures 
d'examen destinées à des groupes d'élèves soumis aux mêmes tests dans des conditions semblables dans tous les pays concernés. Ainsi, avec PISA, l'OCDE produit ses propres données (Henry et al., 2001) tout en obtenant l'accord des différents décideurs politiques qui participent «librement», avec l'appui d'une organisation spécialisée, à cette opération de «surveillance mutuelle» qui prétend améliorer les pratiques de gouvernement (Carvalho, 2014).

Dans le second cas, l'objectif de faire de l'Union Européenne «l'économie de la connaissance la plus compétitive et la plus dynamique du monde» oblige à construire des benchmarks qui servent à orienter tous les États membres. Malgré les différences, nous y retrouvons la même logique de production de connaissances qui va servir notamment les processus de régulation des politiques éducatives: les États membres de l'Union Européenne participent «librement» à des opérations comparées qui se transforment en éléments constitutifs des politiques nationales.

Ces deux cas nous permettent de saisir la logique de comparaison perpétuelle qui s'est installée dans le champ éducatif, logique bien révélatrice non seulement des influences internationales mais aussi des «traductions» qui se produisent lorsqu'elles se transforment en politiques éducatives nationales ou lorsque les organisations internationales réorientent leurs démarches en fonction des processus d'appropriation qui ont cours à l'échelle nationale.

Nous sommes donc face à une réalité nouvelle que l'on commence à peine à pressentir mais qui est fondamentale pour comprendre ce qui se passe dans l'arène des politiques éducatives, sur le plan international et sur le plan des politiques nationales, mais aussi «entre» ces deux espaces d'action. Dans cette nouvelle réalité, et ceci est un point central de notre argumentation, la logique de comparaison n'a pas uniquement des effets «indirects» à travers son incorporation dans les politiques nationales puisque les données sur l'éducation (plus spécifiquement sur la formation et les qualifications) qui sont produites par les processus de comparaison, commencent à migrer, directement, vers les «agrégats d'indicateurs" au même titre que d'autres indicateurs surtout économiques, afin de servir de base aux décisions d'entités internationales, publiques et privées, concernant les différents pays. Dans un monde fortement globalisé, où les pays dépendent de plus en plus des décisions prises par ces «instances» souvent difficiles à identifier et à nommer, l'impact concret des données construites par le biais d'enquêtes comparées en éducation devient tangible.

\section{La lente production d'une logique statistique comparée}

En 1846, Jules Michelet pose une question qui sera reprise, année après année, jusqu'à nos jours: "Quelle est la première partie de la politique? L'éducation. La seconde? L'éducation. Et la troisième? L'éducation» (p. 358). Dans le même 
esprit, Gabriel Compayré, sans doute l'éducateur le plus influent de sa génération à la fin du $19^{\mathrm{e}}$ siècle, écrit dans le Nouveau dictionnaire de pédagogie et d'instruction primaire (1911) que la création des États enseignants est le plus grand événement du $19^{\mathrm{e}}$ siècle, parce que c'est grâce à leur action que se développe l'instruction populaire ou, autrement dit, que s'impose la scolarité obligatoire.

Il faut bien retenir les liens entre l'école de masse ${ }^{1}$ et la construction des États-nations:

L'école de masse est un composant central du modèle de l'État-nation. Sa standardisation collective célèbre la souveraineté unifiée et l'intentionnalité de la collectivité (l'État) [...] et sa culture sécularisée définit le caractère de l'État-nation comme une entreprise qui est conçue pour atteindre le progrès. (Meyer, Ramirez \& Soysal, 1992, p. 131)

La consolidation des États enseignants fait partie d'une rationalité qui est basée sur la croyance illimitée par rapport aux potentialités de l'école. Cette rationalité s'organise sur le plan mondial et l'école devient, tout naturellement, l'un des critères principaux pour établir des «hiérarchies de progrès» entre les pays. Pour ce faire, il devient indispensable de bâtir des dispositifs statistiques en tant qu'outils de régulation des politiques éducatives au niveau national et international.

Il suffit de vérifier l'importance que les rapports statistiques acquièrent au sein des Expositions Universelles de la deuxième moitié du $19^{\mathrm{e}}$ siècle, notamment à partir de l'Exposition de Vienne ${ }^{2}$ en 1873 , pour bien saisir l'ampleur du changement porté par ces outils. G.H. Knibbs écrira que, dorénavant, "les données statistiques remplacent les impressions générales et les destinées des nations seront définies à travers les chiffres» (1910, p. 6). En effet, les indicateurs choisis et les classifications qui émergent ne se bornent pas à décrire la "réalité» de chaque pays. Ils construisent des visions du monde (des différents mondes) et définissent des modèles éducatifs qui influencent, inévitablement, les politiques nationales.

Des recueils statistiques de plus en plus sophistiqués se sont développés tout le long du $20^{\mathrm{e}}$ siècle $^{3}$, intégrant non seulement des données structurelles (nombre d'élèves ou d'enseignants, financement, bâtiments scolaires, etc.), mais aussi des données qualitatives concernant les élèves, le niveau des apprentissages ou les inégalités scolaires. Simultanément, des institutions permanentes de coopération, en particulier le Bureau International d'Éducation, créé en 1925, vont avoir une action importante par rapport au déploiement de logiques de comparaison entre les différents systèmes éducatifs.

Le processus de coopération internationale s'intensifie après la Seconde Guerre Mondiale avec l'expansion d'organisations internationales telles que l'Unesco ou l'OCDE. L'idéologie du "capital humain» va jouer un rôle de plus en plus déterminant dans la construction des politiques éducatives. Les outils de mesure et de comparaison évoluent d'une logique descriptive en direction d'une logique évaluative. Le regard est porté de plus en plus sur les "performances» 
des systèmes éducatifs, notamment sur les indicateurs liés à la qualification des «ressources humaines», à l'emploi et à la compétitivité économique.

Cette longue histoire, de plus d'un siècle, définit quelques éléments de la structure statistique et comparative qui se met historiquement en place pour décrire et mesurer les systèmes éducatifs. Ainsi se produisent et se diffusent, de manière implicite ou explicite, des modèles de référence dans le monde. Aucun pays ne peut s'abstraire de ces «modèles» qui influencent profondément les politiques éducatives de chaque pays, comme l'ont montré les études sur la diffusion mondiale de l'école (voir Nóvoa \& Schriewer, 2000).

Il va sans dire que le processus de traduction sur le plan des États comme celui des tendances définies au niveau international ne sont pas linéaires. Nous sommes confrontés à des logiques très complexes de diffusion et de réception, d'exportation et d'importation, de proposition et d'appropriation, bien expliquées par une littérature très riche dont nous retenons trois auteurs parmi tant d'autres: Jürgen Schriewer, Thomas Popkewitz et Gita Steiner-Khamsi.

Les travaux de Jürgen Schriewer nous invitent à comprendre les processus d'externalisation, notamment en ce qui concerne la manière dont le recours à des «exemples étrangers» sert de légitimation aux dynamiques réformatrices internes à chaque pays. Ces exemples servent de «leçons» fournissant des idées et des appuis nouveaux à la mise en œuvre des politiques éducatives: l'acte de regarder au-delà des frontières, vers des pays comparables (ou vers des «sociétés de référence»), est perçu comme une façon d'acquérir une signification supplémentaire grâce à une logique d'externalisation (c'est-à-dire à une logique d'ouverture vers l'extérieur) (Schriewer, 2000).

Thomas Popkewitz avance des réflexions très intéressantes sur les processus d'indigénisation, notamment sur ce qu'il appelle l'«étranger indigène» (Popkewitz, 2000, 2003). Son argument principal porte sur la manière dont un certain nombre d'idées et de principes ainsi que des individualités ou des noms de référence, sont transportés vers les espaces nationaux comme s'ils étaient des «éléments indigènes». Le travail qu'il a coordonné sur John Dewey et sur la façon dont le "pragmatisme» a voyagé dans le monde est un bon exemple de son approche qui ne cherche pas à analyser la manière dont les réformes internes sont légitimées par l'extérieur, mais plutôt à saisir l'intégration des idées et des auteurs «étrangers» comme s'ils faisaient partie des histoires nationales.

Les recherches menées par Gita Steiner-Khamsi éclaircissent de manière très lucide les processus d'emprunt dans le champ éducationnel. Élaborant sur le jeu du "prêt» et de l'"emprunt», Steiner-Khamsi $(2004,2012)$ se demande comment les acteurs locaux font face et réagissent aux dénommées forces globales: la question n'est pas celle de faire lumière sur les caractéristiques d'un modèle particulier de réforme, mais, au contraire, de valoriser les caractéristiques des "prêteurs» et des «emprunteurs».

Voilà trois processus - externalisation, indigénisation, emprunt - qui se situent du côté de l'«appropriation». Ces auteurs ne contestent pas, bien au 
contraire, l'importance de la "diffusion»; ils cherchent à clarifier la façon dont les modèles globaux se transforment (se métamorphosent) dans les espaces nationaux. Ce déplacement, de la diffusion vers l'appropriation, se situe au cœur des débats sur la traduction.

Notre contribution s'encadre dans ce mouvement de recherche et de pensée. Notre intention est d'attirer l'attention sur un changement qui, pour l'instant, est passé relativement inaperçu. Jusqu'à la fin du $20^{e}$ siècle, le long processus de construction d'outils de comparaison peut être illustré par la métaphore du «miroir». Il s'agit de construire des outils qui permettent de nous regarder en fonction des autres et d'en tirer les conséquences.

Mais aux alentours de l'année 2000, deux événements tendent à transformer cette métaphore et à la remplacer: la réalisation de la première enquête PISA et l'adoption de la Stratégie de Lisbonne. Dorénavant, les logiques de l'examen et du benchmarking introduisent des politiques de traduction qui, petit à petit, se distingueront de celles qui ont marqué le $20^{\mathrm{e}}$ siècle.

\section{PISA 2000: une nouvelle politique de comparaison}

L'histoire de PISA est aujourd'hui bien établie (e.g. Carvalho, 2011, 2014; Morgan, 2007). Le programme fut lancé en 1997 par l'OCDE et les premiers résultats ont été rendus publics en l'an 2001, après la conclusion du premier cycle d'études PISA en 2000. D'après les documents officiels, PISA est une enquête menée tous les trois ans auprès de jeunes de 15 ans dans le but d'évaluer l'acquisition de savoirs et savoir-faire essentiels à la vie quotidienne au terme de la scolarité obligatoire. PISA teste l'aptitude des élèves à appliquer les connaissances acquises à l'école aux situations de la vie réelle, plutôt que la maîtrise d'un programme scolaire précis.

En prenant comme objet l'évaluation les "compétences de littératie des jeunes scolarisés», l'OCDE délimite un domaine propre de connaissances spécialisées, même si le projet est présenté comme étant «un effort collaboratif qui réunit l'expertise scientifique disponible dans les pays participants, dirigé conjointement par les différents gouvernements sur la base d'intérêts partagés et politiquement orientés» (OECD, 2001, p. 3). Au préalable, PISA construit un instrument appliqué aux élèves de tous les pays participants ${ }^{4}$. Cette approche ne traduit pas un changement uniquement méthodologique, le changement est aussi conceptuel et politique. L'approche souligne le caractère régulateur de PISA qui a comme but énoncé d'instaurer de nouveaux dispositifs de légitimation politique et d'aider les gouvernements à devenir plus efficients (Carvalho, 2012).

Le succès de PISA, bien illustré par sa popularité et par son impact dans l'action publique, s'explique par l'existence d'un environnement politique et culturel marqué par une "compétitivité économique globale» où l'éducation et 
les qualifications jouent un rôle central. Dans la perspective de l'OCDE, PISA répond à l'exigence d'aider les gouvernements à prendre leurs décisions politiques en matière d'éducation:

L'objectif de nombreux pays est désormais d'améliorer la gouvernance publique et d'introduire peu à peu une culture d'évaluation. Être à même de comparer les investissements avec les résultats est donc indispensable. En mesurant les performances des élèves, PISA rend possible cette comparaison. (Hugonnier, 2008, p. 48)

PISA n'est pas un simple outil de comparaison. Il établit des manières de penser et d'agir dans les «sociétés basées sur la connaissance». Nous sommes face à de nouvelles conceptions sur l'apprenant et sur l'apprentissage, illustrées par trois discours qui s'entrecroisent: la capacité de chacun à résoudre des problèmes; sa conduite comme entrepreneur et la capacité à se former tout au long de la vie (lifelong learner) pour se maintenir «employable». Ce triple registre caractérise un nouveau répertoire dans la manière de parler et d'agir dans l'espace éducatif.

PISA propose des orientations et induit des comportements: le responsable politique "crédible» et «innovateur» est celui qui gouverne à travers des diagnostics objectifs; c'est celui qui cherche à comprendre les avantages compétitifs à travers la mesure et la comparaison des résultats scolaires et celui qui adopte des solutions basées sur les meilleurs exemples des autres pays. Le responsable politique «moderne» est donc celui qui gouverne à travers un raisonnement comparé et qui accepte la logique de l'«évaluation mutuelle» entérinée par des organismes comme l'OCDE.

Les experts qui dirigent PISA ne se lassent pas de porter des jugements, parfois durs, vis-à-vis des responsables nationaux qui n'adoptent pas ces procédés. Reprenons, à titre d'exemple, une interview d'Andreas Schleicher, chargé de la politique de l'éducation de l'OCDE, à l'un des plus importants magazines brésiliens, Veja:

Le Brésil a commencé à avoir la chance d'avancer à partir du moment où il a commencé à cartographier les problèmes de manière objective - et non plus sur la base de l'intuition des gouvernants. Cela est élémentaire. On ne peut pas penser à améliorer ce qui n'a même pas été mesuré. D'où l'importance de la comparaison internationale. (Weinberg, 2008, pp. 20-21)

L'objectif central de PISA est de contribuer à la «modernisation» des systèmes éducatifs face aux défis de l'économie de la connaissance et de la mondialisation. Cette idéologie permet que les "données PISA» soient lues non seulement dans la dimension espace mais aussi dans la dimension temps. D'une part, ils comparent les différents pays en fonction de leurs résultats, d'autre part, ils construisent, implicitement, une ligne de progrès qui mesure l'évolution de chaque pays vers la «société de la connaissance». Cette double conscience, spatiale et temporelle, est fondamentale pour bien comprendre l'entreprise PISA (Carvalho, 2012). 
La structure centrale de PISA développe toute une série d'activités de production, de médiation et de diffusion de connaissances sur les systèmes éducatifs. Ces connaissances se transmettent au niveau national; les différents acteurs se les approprient et les utilisent de manières très diverses. Nous ne sommes pas devant des logiques linéaires ou simplistes, mais plutôt devant une complexité d'interactions, de réseaux et de légitimités.

À cet égard, il est utile de souligner deux idées:

- Le projet PISA a l'intention de créer une "connaissance utile à la décision politique», ce qui implique, simultanément, la mise en œuvre de dispositifs d'utilisation de cette connaissance par les différents acteurs agissant sur le champ éducatif de chaque pays;

- L'ampleur de cette entreprise oblige à porter une attention spéciale aux questions de la médiation, ce qui implique des consensus qui se construisent entre différents milieux, certainement entre le niveau international et le niveau national mais aussi entre les champs politiques et scientifiques ainsi que dans le rapport entre les sphères spécialisées et l'opinion publique.

Cette analyse de PISA nous conduit directement au concept de traduction, très utile pour comprendre les processus grâce auxquels s'établissent des rapports de coopération entre les mondes cognitifs et sociaux de la recherche et de la politique (Callon, 1986; Latour, 1989).

Le concept de traduction renferme une double acception, linguistique et géométrique: d'une part, la tâche de produire deux versions du même texte dans différents langages entraîne inévitablement des interprétations nouvelles; d'autre part, la tâche de déplacer les textes d'un lieu à l'autre entraîne une réélaboration de significations dans l'espace et dans le temps aussi bien qu'une réinterprétation des objectifs des acteurs sociaux (Latour, 1989).

Voilà ce qui est au cœur de notre argumentation. Les responsables du programme PISA agissent non seulement en tant qu' «agitateurs» ou «instigateurs» mais aussi en tant que "médiateurs» et "constructeurs de réseaux». Ils doivent créer les conditions favorables à la traduction en même temps qu'ils doivent être vigilants vis-à-vis les différentes traductions et appropriations des résultats de leurs enquêtes.

Ce travail est cognitif et social dans la mesure où il produit de la convergence et connecte divers mondes sociaux, mondes qui participent à la production et à la mise en œuvre de tableaux cognitifs et normatifs communs 5 garantissant, en même temps, l'indispensabilité de l'OCDE. Les rapports publiés par l'OCDE à chaque cycle PISA constituent l'un des plus importants produits de ce travail où l'on trouve une logique de traduction qui s'étale sur trois phases principales: d'abord, la révélation, c'est-à-dire l'organisation des tests et des résultats; ensuite, la transformation, c'est-à-dire la lecture des résultats à partir de toute une série de critères dont il faut souligner l'importance de l'axe "efficience versus équité» et celui des facteurs de contextualisation; finalement, la condensation, c'est-à-dire 
l'élaboration de recommandations et d'orientations adressées aux décideurs politiques (Carvalho, 2012).

Mais le plus important va se passer dans la traduction de ces documents dans les politiques nationales, ce qui exige des processus complexes d'appropriation et de réélaboration. Aujourd'hui - et c'est là l'essentiel de notre argumentation - ces processus de traduction entraînent des conséquences significatives dans la vie des États du point de vue matériel et pas uniquement du point de vue symbolique.

Dans le passé, les statistiques et les tableaux comparés constituaient des facteurs de prestige et représentaient, sans doute, un «capital» important non seulement pour la place que chacun était tenu d'occuper dans le monde mais aussi pour les rapports de force entre les différents pays. Cela avait des conséquences non négligeables mais ces conséquences étaient, d'une certaine manière, indirectes. Elles portaient sur des facteurs de prestige et tenaient compte de la façon dont les orientations des «instances internationales» étaient traduites au plan national (Bos \& Schwippert, 2009).

Le changement majeur qui est en train de se produire depuis quelques années et est encore très difficile à cerner, passe par la reconnaissance de l'existence des conséquences bien plus directes, concrètes, des résultats de ces grandes enquêtes et classifications internationales. Dans un monde fortement globalisé, les décisions internationales concernant des prêts financiers ou la notation des pays par des agences internationales, avec tous les effets que l'on connait, ou encore les options d'investissement économique sont directement liées à des aspects comme la qualification des "ressources humaines», la qualité des systèmes éducatifs ou les apprentissages des élèves tels qu'ils sont définis par des programmes comme PISA.

Il suffit de mentionner l'importance que la Banque Mondiale ou le Fonds Monétaire International accordent, dans leurs analyses, aux dimensions concernant l'éducation, la formation et l'employabilité. Ces indicateurs servent à établir des classements qui sont utilisés au moment de prendre des décisions concernant l'aide financière ou la façon de dessiner les programmes de financement des différents pays. Les documents du Forum Économique Mondial sont aussi très éclairants. Retenons, par exemple, The Global Competitiveness Report 2013-2014 qui a des implications immédiates par rapport aux décisions officielles ou privées de soutien ou d'investissement dans certains pays. Ce rapport inclut toute une série de données concernant l'éducation: qualité de l'éducation primaire, taux de scolarisation, qualité du système éducatif, qualité de l'éducation en sciences et mathématiques, qualité de la gestion des écoles, etc. (Schwab, 2013).

Aussi, des agences de notation, telles que Moody's ou Standard and Poor's, tendent à incorporer, dans leurs rapports, des données provenant des grandes enquêtes éducationnelles. Cet argument est développé de façon très intéressante par Susan Robertson dans son analyse des rankings internationaux des 
universités, quand elle explique l'utilisation que les agences de notation font de plusieurs données et classements: "Ces utilisations suggèrent que ces rankings sont en train d'être utilisés de façons qui vont bien au-delà des 'instructions' affichées dans les étiquettes des produits” (Robertson, 2012, p. 245).

PISA fait partie d'un processus qui sert à légitimer la croyance d'après laquelle le changement éducationnel peut conduire les nations au "nouveau» monde global de la «société de la connaissance». Or, cette dimension fictionnelle est politiquement très matérielle. Nous sommes devant un outil de régulation qui agit par la qualification et la disqualification des acteurs politiques, par la culpabilité et l'espoir qu'il crée.

Voilà qui attribue à ces instruments un nouveau rôle dans la coopération internationale mais surtout dans la régulation des politiques éducatives nationales. Nous nous trouvons face à un changement que l'on commence à peine à identifier mais qui va influencer profondément les politiques et les sociétés du $21^{\mathrm{e}}$ siècle.

\section{Union Européenne 2000: Les conséquences de La comparaison}

L'intervention des instances européennes dans le domaine de l'éducation a été fortement contestée pendant longtemps, tous les traités stipulant la nécessité de respecter «pleinement la responsabilité des États membres pour le contenu de l'enseignement et l'organisation de son système éducatif» (article 149 du Traité d'Amsterdam). Et pourtant aujourd'hui, nul ne doute que des initiatives sont prises, depuis longtemps, dans le but d'inscrire une dimension européenne dans les politiques nationales d'éducation. Il faut retenir deux dates: le Traité de Maastricht (1992) et le Sommet de Lisbonne (2000) (Nóvoa \& Lawn, 2002).

Progressivement, le discours européen tend à s'imposer comme idéal régulateur, définissant des "possibilités» et des «impossibilités» dans le champ de l'éducation. En 2001, Anders Hingel, à l'époque chef de l'unité des politiques éducatives à Bruxelles, explique que:

Les conclusions de Lisbonne ont accordé à l'Union, implicitement, un mandat pour développer une approche commune dans le domaine de l'éducation. Ce mandat est bien clair dans la demande formulée auprès des ministres pour débattre les objectifs communs de leurs systèmes éducatifs. Voilà ce qui conduira à un renforcement de la dimension européenne des politiques éducatives nationales. (2001, p. 19)

Le changement, très important, est justifié par les décisions prises au Sommet de Lisbonne en 2000. Celles-ci concernent l'adoption d'une nouvelle méthode ouverte de coordination et l'ambition de faire entrer - en la transformant l'Union Européenne dans «l'économie de la connaissance la plus compétitive et la plus dynamique au monde». 
Ainsi, c'est sans surprise que l'Union Européenne élabore, en 2001, un document essentiel - Les objectifs concrets futurs des systèmes d'éducation - qui prétend développer «une approche cohérente de l'action menée au niveau communautaire» et organiser "un cadre structuré où les discussions et les actions politiques puissent s'inscrire au cours des prochaines années» afin de réussir "un renforcement de l'impact politique de la coopération communautaire» (Commission Européenne, 2001, p. 16).

Les initiatives politiques européennes s'organisent autour du programme Éducation \& Formation 2010 qui fait ressortir deux grands thèmes autour desquels se tissent les arguments centraux. Le premier concerne la nouvelle société (ou économie) basée sur la connaissance et les principes de compétitivité et d'esprit entrepreneurial. Le second a trait aux questions de l'apprentissage et de la façon dont elles sont successivement mobilisées, notamment à partir des références à l'employabilité et à l'éducation et formation tout au long de la vie.

Il y a quatre verbes (d'action) qui organisent le programme Éducation \& Formation 2010 et qui constituent des analyseurs de la manière dont les politiques éducatives européennes sont traduites au niveau de chaque État membre: identifier (des objectifs communs); diffuser (les bonnes pratiques); mesurer (les résultats obtenus); comparer (les évolutions et les politiques d'un pays à l'autre). Ces quatre verbes définissent les frontières d'une démarche d'action politique qui repose toujours sur la méthode ouverte de coordination.

Malgré l'échec du programme Éducation \& Formation 2010 qui n'a pratiquement pas atteint un seul de ses objectifs, l'Union Européenne a pris la décision de lancer une nouvelle stratégie, Europe 2020, qui, à beaucoup d'égards, répète les mêmes logiques et manières d'agir.

Construite autour de cinq grands agrégats d'indicateurs, Europe 2020 consacre le premier objectif à l'emploi, le troisième au climat et à l'énergie et le cinquième à la réduction de la pauvreté. Ce sont trois objectifs qui concernent et mobilisent les politiques éducatives, sur le plan social et sur celui de la recherche. Mais ce sont le deuxième et le quatrième objectifs qui marquent les enjeux explicites des systèmes d'enseignement général et supérieur: le deuxième objectif est centré sur l'investissement en science (recherche \& développement); le quatrième établit deux buts quantifiables, à savoir moins de $10 \%$ de taux d'abandon scolaire et plus de $40 \%$ de jeunes d'une même génération ayant obtenu un diplôme d'enseignement supérieur d'ici 2020.

Ce qui est encore plus important: ces objectifs ne correspondent pas à des moyennes européennes mais ils doivent être obligatoirement atteints par chaque État membre. Pour ce faire, l'Union Européenne mettra en place un système d'accompagnement, de contrôle et de vérification et établira des rapports de progrès et des benchmarks de manière à pouvoir suivre soigneusement les évolutions internes de chaque pays (Lawn \& Nóvoa, 2013).

À travers la construction d'indicateurs librement acceptés et agréés par tous les pays à travers des processus d'échange de bonnes pratiques librement adoptés 
par chaque État membre, l'Union Européenne met en place des politiques basées sur des logiques de comparaison, des logiques qui sont en train de devenir l'un des moyens les plus puissants de l'éducation.

Il y a, d'une part, une sorte de processus de "gouverner sans gouverner», c'està-dire de gouverner comme s'il n'y avait pas de prise de décisions politiques, comme s'il s'agissait d'établir une politique éducative sans avoir besoin de la formuler explicitement (Nóvoa, 2010). Il y a, d'autre part, un processus de "gouverner à travers les chiffres» (Ozga, 2009), comme s'il s'agissait uniquement de comparer et de confronter des chiffres, des éléments «neutres», des mesures «objectives».

De manière simplifiée, on pourrait dire que la comparaison est en train de devenir La politique, ce qui entraine des processus permanents de traduction de la sphère européenne vers les sphères nationales. Relevons que ces processus, tels que nous venons de les exposer, sont bien établis et ont été finement analysés au niveau de l'espace européen de l'éducation (Lawn \& Nóvoa, 2013).

Cependant, dans le cadre de la stratégie Europe 2020, il est un changement observable qui n'a pas encore reçu toute l'attention qu'il mérite: les données sur l'éducation commencent à intégrer des ensembles d'indicateurs qui cherchent à définir la «santé» des pays. Ces données ont des conséquences concrètes sur les politiques européennes notamment en ce qui concerne la définition de pré-conditions pour les appuis financiers, pour l'attribution de fonds ou même pour l'éligibilité et l'accès à certains programmes communautaires.

Ainsi, sur le fond de la crise économique européenne, les différents indicateurs sont intégrés de telle manière qu'ils constituent un référentiel pour les prises de décision concernant les politiques vis-à-vis de chaque État membre. Retenons, à titre d'exemple, l'importance qu'acquiert l'«examen annuel de la croissance», examen qui fixe les priorités de l'Union européenne tout en définissant des recommandations pour chaque pays. Dorénavant, il ne s'agit plus de systématiser les données afin que chaque pays en tire les conséquences utiles mais il s'agit, bien au contraire, de définir des orientations que chaque pays doit suivre. Autrement dit, il s'agit de circonscrire ce que chaque pays doit "obligatoirement» traduire. Et chaque État membre, dans sa «liberté», doit «inévitablement» adopter ces recommandations.

De manière implicite, c'est ce que le Président de la Commission Européenne, José Manuel Barroso, écrit dans la préface au document Europe 2020:

2010 doit marquer le début d'une nouvelle ère, au cours de laquelle je souhaite voir l'Europe sortir renforcée de la crise économique et financière. (...) Nous devons admettre que l'interdépendance accrue de nos économies impose de réagir de manière plus résolue et plus cohérente au niveau politique. (Commission Européenne, 2010, p. 2) 
Le document de présentation de l'examen annuel de croissance explique que les orientations définies doivent être incorporées dans les programmes de réforme à entreprendre par chaque État membre, ainsi que dans les décisions économiques et budgétaires nationales. Or - et cela mérite d'être souligné l'évaluation à entreprendre intègre aussi des indicateurs éducatifs, notamment ceux qui ont trait aux qualifications et à l'employabilité.

Il suffit de rappeler les accords signés dans le cadre des programmes d'assistance financière de l'Union Européenne à la Grèce, à l'Irlande et au Portugal. Ces accords définissent, de manière assez détaillée, les politiques que ces pays doivent conduire pour continuer à bénéficier de l'appui de l'Union. Les questions budgétaires, économiques et financières, sont centrales mais on ne peut pas ignorer l'importance octroyée à d'autres secteurs, notamment le secteur éducatif. Ainsi, chaque "évaluation" des programmes d'assistance est associée à des orientations qui sont adressées, non pas comme des «suggestions» mais comme des "orientations à suivre». Celles-ci s'organisent autour de thèmes tels que la qualité de l'enseignement, l'employabilité, l'éducation et la formation tout au long de la vie, le renforcement de la formation professionnelle et l'accroissement de la participation des entreprises aux systèmes d'enseignement et de formation. Ces orientations se tissent à partir de données comparées et des résultats des grandes enquêtes internationales sur l'éducation ${ }^{6}$.

Dans le cadre de la stratégie Europe 2020, l'Union Européenne lance un nouveau programme appelé Repenser l'éducation au sous-titre lourd de significations - «Investir dans les compétences pour de meilleurs résultats socioéconomiques»:

Les systèmes d'éducation et de formation européens ne forment toujours pas aux compétences pertinentes pour l'employabilité et ne collaborent pas suffisamment avec les entreprises ou les employeurs pour faire converger le processus d'apprentissage et la réalité professionnelle. (Commission Européenne, 2012)

Ce passage rend bien compte de l'ambiance du document porté par la crise économique et inspiré par des politiques éducatives qui doivent répondre aux besoins de redressement de l'économie. Dans le champ éducatif, le processus d' «unionisation» se concrétise à travers l'adoption d'orientations qui mettent l'accent sur les thèmes de l'employabilité et de la formation tout au long de la vie. Dorénavant, comme nous avons essayé de le démontrer, la traduction de ces orientations au plan national ne renferme pas uniquement un jeu d'influences mais elle entraîne des conséquences concrètes pour chaque État membre.

\section{Notes conclusives: une traduction lourde de conséquences}

Les analyses relatives aux changements dans les modes de gouvernement de l'éducation révèlent que la production et la gestion des politiques publiques 
dépendent de la mobilisation d'une connaissance spécialisée et de l'intervention des agences internationales. Tout le long de ce texte nous avons analysé deux de ces réalités: le programme PISA, sous la responsabilité de l'OCDE, qui est en train de redéfinir l'espace des politiques éducatives et l'action de l'Union Européenne dans le cadre de l'éducation et les processus de confluence qu'elle produit.

Il y a certes beaucoup de différences entre ces deux réalités. La principale, bien sûr, a trait au statut même de l'OCDE et de l'Union Européenne car, dans ce dernier cas, nous sommes devant un projet politique de coopération et d'intégration. Mais les similarités sont nombreuses, comme nous l'avons argumenté, surtout par rapport à la manière de produire une connaissance spécialisée, de systématiser cette connaissance en divers indicateurs entraînant la montée de logiques de mesure et de comparaison entre pays, par rapport à la manière de traduire cette comparaison en politiques éducatives nationales et, finalement, d'utiliser ces données comparées - ce qui a été réalisé surtout au cours de la dernière décennie - comme matériaux ayant des conséquences concrètes sur les politiques économiques, sociales et éducatives des différents pays.

Ce sont des processus qui s'enchaînent sans aucune linéarité et qui s'organisent au sein d'un amalgame, d'un assemblage et de la juxtaposition de réseaux, de nœuds et d'influences parfois conflictuels. Des instances diverses agissent aux frontières de la connaissance et de l'action publique, aux frontières de différents niveaux de décision politique, entre espaces nationaux et internationaux.

Le caractère frontalier est essentiel pour bien comprendre la recomposition des significations et des dispositifs de traduction qui sont mis en place de façon systématique et permanente. C'est bien ce caractère qui permet les rencontres entre différents groupes et instances qui produisent "les instruments d'action publique» (Lascoumes \& Le Galès, 2007). C'est pourquoi il est nécessaire de maintenir une tension interprétative entre deux perspectives qui ne s'excluent pas mutuellement, même si, à première vue, elles peuvent paraître contradictoires.

Il s'agit, d'une part, de souligner la vision "polycentrique» du pouvoir et la spécificité des traductions qui ont lieu dans les différents espaces politiques. Il faut éviter de reproduire des images d'un monde artificiellement unifié car il y a toujours des processus de localisation des politiques, ne serait-ce qu'en raison des contextes et des circonstances culturelles et historiques spécifiques (Popkewitz, 2000). Nous ne pouvons pas comprendre une "politique éducative itinérante» (Ozga \& Jones, 2006), élaborée au sein d'instances internationales, sans identifier les processus de médiation, de réinterprétation, de négociation et de contextualisation qui accompagnent la réception ainsi que la façon dont ces «voyageurs» cherchent à s'adapter aux cultures et aux structures des espaces nationaux locaux.

Il s'agit, d'autre part, de bien marquer l'émergence de manières de penser et d'agir dans le champ éducatif, manières qui tendent à unifier notre répertoire de possibilités. À cet égard, il faut noter l'émergence d'une «nouvelle langue planétaire» qui tend à uniformiser différentes langues éducatives. Des notions comme 
société de la connaissance, employabilité ou formation tout au long de la vie sont devenues, partout, des composantes inévitables des discours et des politiques éducatives. Ils définissent les contours d'un discours spécialisé mais ils orientent aussi la façon dont l'opinion publique prend conscience des thèmes liés à l'éducation et de leur importance politique.

Face à ces deux perspectives, il faut développer des instruments de pensée critique qui permettent d'élargir nos possibilités d'analyse et de réflexion. C'est là le point central du travail scientifique: élargir le répertoire des manières de penser et de parler dans le champ éducatif.

C'est bien ce que nous avons essayé de produire, attirant l'attention sur le changement qui est en train d'avoir lieu à partir du développement des approches comparées, notamment de celles qui sont conduites par l'OCDE à travers le programme PISA et par l'Union Européenne dans le cadre des politiques postérieures à l'adoption de la Stratégie de Lisbonne en l'an 2000.

La compréhension de ce changement est essentielle à l'éclaircissement des politiques éducatives et à la compréhension de l'ensemble complexe des interactions, des influences et des traductions qui les caractérisent sur les plans international, national et local. Nous sommes bien conscients du fait que le tournant que nous avons essayé de saisir est encore difficile à cerner et à argumenter à travers du «matériel empirique». Mais il est certainement déjà présent, discernable à l'analyse de la manière dont les données comparées en éducation sont en train d'être utilisées dans différentes arènes politiques. C'est pourquoi nous avons voulu prendre le risque d'avancer dans cette compréhension en attirant l'attention sur les nouvelles logiques de traduction qui, d'après nous, vont se développer dans les années à venir.

\section{Notes}

1 Nous utilisons le concept d'école de masse pour signifier ce que nos collègues anglo-saxons appellent «mass schooling», tout en reconnaissant que la traduction est imparfaite. Il s'agit d'identifier le processus d'imposition de l'obligation scolaire, dans la transition du $19^{\mathrm{e}}$ vers le $20^{e}$ siècle. À ne pas confondre avec le concept de massification de l'enseignement, processus bien plus tardif, de la deuxième moitié du $20^{\mathrm{e}}$ siècle.

2 Deux longs rapports présentés à Vienne méritent une attention particulière: le Rapport sur l'instruction primaire à l'Exposition Universelle de Vienne en 1873 par Ferdinand Buisson et le Rapport sur l'instruction primaire et l'instruction secondaire par Émile Levasseur.

3 Voir, par exemple, les Annuaires de l'Éducation publiés par le Teachers College à New York depuis 1924, The Year Book of Education diffusé par l'Institut de l'Éducation à Londres après 1932 ou l'Annuaire International de l'Éducation et de l'Enseignement que le Bureau International d'Éducation fera sortir dès 1933.

4 Comme règle générale, dans chaque pays sont examinés entre 4'500 et 10 ’000 élèves.

5 Par exemple, sur ce que doit être l'éducation au $21^{\mathrm{e}}$ siècle, sur ce qui définit les "compétences» d'un jeune scolarisé et la manière de les appréhender ou encore sur les modalités les plus efficientes pour gouverner les systèmes éducatifs.

6 Le site web de l'Union Européenne propose de nombreux documents concernant les programmes d'assistance financière aux trois pays mentionnés. 


\section{Références bibliographiques}

Bos, W. \& Schwippert, K. (2009). TIMSS, PISA, IGLU y demás: razón y sinrazón de los estudios internacionales de rendimiento escolar. Profesorado - Revista de currículum y Formación del profesorado, 13 (2), 1-15.

Callon, M. (1986). Some Elements of a Sociology of Translation. In J. Law (Éd.), Power, Action and Belief: A New Sociology of Knowledge (pp. 196-233). London: Routledge \& Kegan Paul.

Carvalho, L. M. (2011). Look at the Mirror! On the cognitive and normative features of a knowledge-policy tool. In J. Kusch (Éd.), Knowledge, Differences and Harmonies in the Time of Globalisation (pp. 61-78). Cambridge: Cambridge Scholars Publishing.

Carvalho, L. M. (2012). The fabrications and travels of a knowledge-policy instrument. European Educational Research Journal, 11 (2), 172-188.

Carvalho, L. M. (2014). The attraction of mutual-surveillance of performances: PISA as a knowledge-policy instrument. In T. Fenwick, J. Ozga \& E. Mangez (Éd.), World Yearbook of Education 2014: Governing Knowledge: Comparison, Knowledge-based Technologies and Expertise in the Regulation of Education (pp. 58-72). Oxford: Falmer/Routledge.

Commission Européenne (2001). Les objectifs concrets futurs des systèmes d'éducation. Bruxelles: $\operatorname{COM}(2001) 59$ final.

Commission Européenne (2010). Communication de la Commission: Europe 2020 - Une stratégie pour une croissance intelligente, durable et inclusive. Bruxelles: COM(2010) 2020 final.

Commission Européenne (2012). Communication de la Commission: Repenser l'éducation - Investir dans les compétences pour de meilleurs résultats socioéconomiques. Bruxelles: $\operatorname{COM}(2012) 669$ final.

Compayré, G. (1911). Pédagogie (Histoire de la). In F. Buisson (Éd.), Nouveau dictionnaire de pédagogie et d'instruction primaire (pp. 1546-1550). Paris: Librairie Hachette et Cie.

Henry, M., Lingard, B., Rizvi, F. \& Taylor, S. (2001). The OECD globalisation and education policy. Oxford: Pergamon Press.

Hingel, A. (2001). Education policies and European governance. Brussels: European Commission - Directorate-General for Education and Culture.

Hugonnier, B. (2008). Les acquis des adolescents. Futuribles, 344, 47-61.

Knibbs, G.H. (1910). The Evolution and Significance of the Census. Melbourne: Imperial Federation League of Australia.

Lascoumes, P. \& Le Galès, P. (2007). Introduction: understanding public policy through its instruments. Governance, 20 (1), 1-21.

Latour, B. (1989). La science en action. Paris: La Découverte.

Lawn, M. \& Nóvoa, A. (2013). Introduction: The European Educational Journal - New fabrications. Sisyphus - Journal of Education, 1 (1), 11-17.

Meyer, J., Ramirez, F. \& Soysal, Y. (1992). World expansion of mass education, 1870-1980. Sociology of Education, 65 (2), 128-149.

Michelet, J. (1846). Le Peuple. Paris: Hachette / Paulin.

Morgan, Clara (2007). The OECD Programme for International Student Assessment: Unraveling a Knowledge Network. Ottawa, Ontario: Doctoral Thesis, School of Public Policy and Administration, Carleton University.

Nóvoa, A. (2010). Governing without governing - The formation of a European educational space. In M.W. Apple, S.J. Ball \& L.A. Gandin (Éd.), The Routledge International Handbook of the Sociology of Education (pp. 264-273). Oxon: Routledge,.

Nóvoa, A. \& Lawn, M. (Éd.). (2002). Fabricating Europe - The formation of an education space. Dordrecht: Kluwer Academic Publishers.

Nóvoa, A. \& Schriewer, J. (Éd.). (2000). A difusão mundial da escola: Alunos, Professores, Currículo, Pedagogia. Lisboa: Educa. 
Nóvoa, A. \& Yariv-Mashal, T. (2003). Comparative Research in Education: a mode of governance or a historical journey? Comparative Education, 39 (4), 423-438.

OECD (2001). Knowledge and skills for life - First results from PISA 2000. Paris: OECD.

Ozga, J. (2009). Governing Education through Data in England: From Regulation to Self-Evaluation. Journal of Education Policy, 24 (2), 149-163

Ozga, J. \& Jones, R. (2006). Travelling and embedded policy: the case of knowledge transfer. Journal of Education Policy, 21 (1), 1-17.

Popkewitz, T.S. (2000). Globalisation/Regionalisation, knowledge, and the educational practices. In T.S. Popkewitz (Éd.), Educational Knowledge: Changing relations between the state, civil society, and the educational community (pp. 3-27). New York: State University of New York Press.

Popkewitz, T.S. (2003). National imageries, the indigenous foreigner, and power. In J. Schriewer (Éd.), Discourse Formation in Comparative Education (pp. 261-294). Frankfurt, Berne: Peter Lang.

Popkewitz, T.S. (Éd.). (2005). Inventing the Modern Self and John Dewey: Modernities and the Traveling of Pragmatism in Education. New York: Palgrave/Macmillan.

Robertson, S. (2012). World-class higher education (for whom?). Prospects, vol. 42, 237-245.

Schleicher, A. (2006). Fundamentos y cuestiones políticas subyacentes al desarrollo de PISA. Revista de Educación, numéro spécial sur PISA, 21-43.

Schriewer, J. (2000). World-system and interrelationship-networks. In T.S. Popkewitz (Éd.), Educational Knowledge: Changing relations between the state, civil society, and the educational community (pp. 305-342). New York: State University of New York Press,.

Schwab, Klaus, ed. (2013). The Global Competitiveness Report 2013-2014. Geneva: World Economic Forum.

Steiner-Khamsi, G. (2004). The Global Politics of Educational Borrowing and Lending. New York: Teachers College Press.

Steiner-Khamsi, G. (2012). Understanding policy borrowing and lending: building comparative policy studies. In G. Steiner-Khamsi \& F. Waldow (Ëd.), World Yearbook of Education 2012: Policy Borrowing and Lending in Education (pp. 3-17). London: Routledge.

Weinberg, M. (2008). Entrevista: Andreas Schleicher - Medir para avançar rápido. Veja, 17 août 2008, 20-21.

Mots-clés: Comparaison, espace européen de l'éducation, gouvernance, PISA, statistique comparée.

\section{International vergleichende Leistungsstudien und Bildungspolitik: Wendepunkt anfangs des 21. Jahrhunderts}

\section{Zusammenfassung}

Unser Text versteht sich als Essay, der in freier Form eher versucht, eine Diskussion über Ideen zu eröffnen, als Thesen empirisch zu belegen. Das dabei vorgebrachte Hauptargument betrifft das Auftreten einer Reihe bedeutender Veränderungen, wie etwa die «internationalen und vergleichenden Leistungsstudien», die Einzug in die nationale Bildungspolitik verschiedener Staaten gehalten haben. Dies geschah ab 2000, einem Zeitpunkt, den wir für symbolisch halten. Es soll, mit anderen Worten, dargestellt werden, wie solche Studien zu 
einer «Übersetzungspolitik» geführt haben, die sich deutlich von der für das 20 . Jahrhundert charakteristischen Politik unterscheidet.

Schlagworte: Vergleich, europäischer Erziehungs- und Bildungsraum, Gouvernance, PISA, vergleichende Statistik.

\section{II confronto come politica: la svolta all'inizio del XXI secolo}

Riassunto

Il nostro testo costituisce un tentativo libero di aprire un dibattito d'idee piuttosto che di dimostrare empiricamente determinate tesi. L'argomento centrale sviluppato nello stesso riguarda l'emergenza - dall'anno 2000, che abbiamo stabilito come data simbolica, - di tutta una serie di cambiamenti maggiori riguardo al modo in cui $\mathrm{i}$ "dati internazionali e comparati» sono incorporati nelle politiche educative nazionali o, in altre parole, sulla maniera in cui tali dati inducono politiche di traduzione che si distinguono nettamente da quelle che hanno caratterizzato il $20^{\circ}$ secolo.

Parole chiave: Confronto, spazio europeo dell'educazione, governo, PISA, statistica comparata.

\section{Comparison as politics: The turning point of the early 21th century}

\section{Summary}

This contribution is an open essay that aims at opening a debate, rather than demonstrating certain theses by means of empirical evidence. We mainly argue that from the year 2000 on, a series of important changes occurs regarding the way international comparative data are incorporated within national educational politics or, in other words, with respect to the way these data yield translation politics clearly different from those featuring the 20th century.

Key words: International comparison, comparative statistics, European education, educational politics, PISA. 